\title{
Defining the Critical Elements of the Most Common Arthroscopic Procedures: A Consensus of Orthopaedic Sports Medicine Surgeons
}

David A. Porter ${ }^{1}$, Joseph L. Laratta ${ }^{2}$, Jamal N. Shillingford ${ }^{3}$, David Trofa ${ }^{4}$, Hemant Reddy ${ }^{5}$, John W. Uribe $^{1}$, Gautam P. Yagnik ${ }^{1}$

1. Orthopaedics, Baptist Health South Florida, Coral Gables, USA 2. Orthopaedics, Norton Leatherman Spine Center, Louisville, USA 3. Orthopedics, Norton Leatherman Spine Center, Louisville, USA 4. Orthopedics, Columbia University Medical, New York, USA 5. Orthopaedics, Northeast Ohio Medical University (NEOMED), Rootstown, USA

Corresponding author: Hemant Reddy, hreddy2@neomed.edu

\section{Abstract}

\section{Objective}

To define the critical elements of common procedures in arthroscopic surgery.

\section{Methods}

A survey was administered to surgeons associated with the American Orthopaedic Society for Sports Medicine (AOSSM) to determine the critical elements for four common arthroscopic procedures: anterior cruciate ligament (ACL) reconstruction, knee arthroscopy with meniscal debridement or repair, rotator cuff repair (RCR), and capsulorrhaphy for anterior glenohumeral instability (Bankart repair). Respondents were asked which steps necessitated their direct supervision. The level of experience and practice demographics were also recorded.

\section{Results}

For all applicable procedures, patient positioning and closure were not considered critical steps. Establishing arthroscopic portals was critical for all procedures, except knee arthroscopy. Diagnostic arthroscopy was only critical in ACL reconstruction. Private practice surgeons considered every step of these common procedures to be critical elements. Less experienced surgeons were more likely to regard certain aspects of a procedure critical. Surgeons with $>15$ years of experience considered diagnostic arthroscopy critical to all procedures, whereas those with $<15$ years of experience did not. Unlike surgeons with a resident as first assist, surgeons with a physician assistant (PA) or nurse practitioner (NP) found every step of each procedure to be critical except closure and positioning.

\section{Conclusion}

Received 11/05/2018

Review began $11 / 25 / 2018$ Review ended 02/13/2019 Published 02/19/2019

\section{() Copyright 2019}

Porter et al. This is an open access article distributed under the terms of the Creative Commons Attribution License CC-BY 3.0., which permits unrestricted use, distribution, and reproduction in any medium, provided the original author and source are credited.
Across all procedures, only patient positioning and closure were consistently regarded as non-critical elements. There were significant differences in responses according to experience and practice setting. Future research is necessary to determine the implications of these findings and guide the definition of the “critical portions" of surgery.

\section{Categories: Orthopedics}

Keywords: sports medicine, arthroscopy, surgery, critical

\section{Introduction}

Every operation is a sequence of numerous, distinct steps that carry their own potential for complications. Academic teaching hospitals train resident surgeons by allowing them to assist in these operations and take an increasingly important role as both their knowledge and skills mature. The notion of "critical” steps of surgery, or those requiring the presence and direct supervision of the attending surgeon, has been implemented into nearly every document pertaining to surgery, from patient consent forms to national guidelines, and even billing clauses [1-2]. Recently, the Boston Globe's investigation on the practice of concurrent surgeries drew controversy over "double booking” - the process of overlapping surgical cases booked under one attending surgeon in multiple rooms [1,3]. Questions of patient safety, ethical consent, health care costs, and medical education and training have arisen from this investigation despite the clear language in patient consent forms that reassures patients that an attending surgeon will be present for all "critical parts" of the procedure.

In April 2016, the American College of Surgeons' (ACS') Statement of Principles was revised and distinguishes between "concurrent" and "overlapping” surgeries based on whether the "critical steps" of the two procedures occur simultaneously or sequentially [4]. This statement deems concurrent surgeries 
inappropriate, as they do not allow the presence of an attending surgeon during the "critical" steps of surgery occurring simultaneously. The Center for Medicare and Medicaid Services (CMS) has also declared that a supervising physician must be present for all "critical portions" of a procedure in order to qualify for reimbursement [2]. While the language in these consent forms is consistent across academic centers, this notion of "critical parts" of surgery still remains unclear, as it is open to various interpretations. To date, there has been no study establishing criteria for critical surgical steps in arthroscopic surgery, although it has been implied that such steps are "defined" for all common surgical procedures [5].

The purpose of the current study was to define the critical elements in four of the most common arthroscopic sports medicine procedures: anterior cruciate ligament (ACL) reconstruction, rotator cuff repair (RCR), knee arthroscopy, and anterior capsulorrhaphy with labral repair for glenohumeral instability (Bankart procedure). Herein, we report the results of a survey distributed to orthopedic sports medicine surgeons. We hypothesized that for each procedure, patient positioning, portal establishment, diagnostic arthroscopy, and closure would not be considered critical steps.

\section{Materials And Methods}

A web-based survey was developed by the authors at our institution and administered using Qualtrics software (Provo, UT, US), a survey platform for online data collection and analysis. The questionnaire consisted of 11 questions. The first seven questions were directed at surgeon demographics (years in practice, private versus academics, urban versus rural, geographic location, fellowship training, and first assistant). Each of the final four questions focused on a particular procedure, specifically ACL

reconstruction, RCR, knee arthroscopy with meniscal treatment, and Bankart procedures. The order of the procedures was randomized for each respondent. Each of the procedures was separated into its constituent elements, including the positioning, portal placement, diagnostic arthroscopy, closure, and major steps in each respective procedure. The following statement headed each of these questions: "Which are the "critical" elements of surgery requiring your direct supervision in the operating room? Check ALL boxes that apply." Respondents selected the portions of each procedure that necessitate their direct supervision in the operating room. If any of the major aspects of the procedure were selected by respondents, a sub-menu appeared with further questions. For the purpose of this study, a "critical" element of arthroscopic surgery was defined as a surgical step selected by the majority of respondents (>50\%).

A link to the survey was emailed to 2016 members of the American Orthopaedic Society for Sports Medicine (AOSSM). Between August and September 2017, 343 physicians responded to the survey, corresponding to a response rate of $17 \%(343 / 2016)$.

Statistical analyses were performed with SPSS (IBM v24.0, Chicago, IL, US). An unadjusted univariate analysis was performed using independent sample t-tests for continuous data and Chi-squared or Fisher exact tests for categorical variables. Correlations between continuous variables were examined using the Pearson correlation coefficient test. Statistical significance was defined as $\mathrm{p}<0.05$.

\section{Results}

A total of 343 surgeons responded to the survey. Responder demographics are presented in Table 1 . The majority of respondents practiced in a major city (55.9\%) with over 15 years of surgical experience (55\%). Among respondents, the first assistant during surgery was a resident physician (30\%), a physician assistant (PA) (45.9\%), a nurse practitioner (NP) (4.1\%), or other (20\%). For all procedures, patient positioning and closure were not designated as critical elements. Diagnostic arthroscopy was not considered critical in any procedure, except for ACL reconstruction. The establishment of portals was a critical element of all procedures except knee arthroscopy.

\section{All Responders (\%)}

No. of Attending Surgeons

$343(17)$

Years in Practice, $\mathrm{n}(\%), \mathrm{N}=340$

1-5 Years

30 (8.8)

6-10 Years

$85(25.0)$

11-15 Years

38 (11.2)

$15+$ Years

$187(55.0)$

Sports Medicine/Shoulder Fellowship Completed, n (\%), N=341

Sports Medicine

306 (89.7)

Shoulder

7 (2.1) 


\section{Cureus}

Neither

28 (8.2)

Practice Classification, $n$ (\%), $\mathrm{N}=341$

Academic

$83(24.3)$

Private

$183(53.7)$

Privademic

$75(22.0)$

Practice Location, n (\%), N=340

Northeast

$102(30.0)$

South

77 (22.6)

West

40 (11.8)

Central

$85(25.0)$

Southwest

29 (8.5)

Outside of USA

7 (2.1)

Practice Location Classification, $n$ (\%), N=340

Major City

190 (55.9)

Minor City

$130(38.9)$

Rural

20 (5.9)

Cases Performed Each Year, $\mathrm{n}(\%), \mathrm{N}=341$

$<100$

$6(1.8)$

$100-250$

68 (19.9)

250-500

$186(54.5)$

$500-700$

57 (16.7)

$>700$

$24(7.0)$

First Assistant During Surgery, n (\%), N=340

Resident (MD/DO)

$102(30.0)$

Physician Assistant

$156(45.9)$

Nurse Practitioner

$14(4.1)$

Other

$68(20.0)$

\section{TABLE 1: Responder demographics}

\section{Among all respondents}

For knee arthroscopy, meniscal debridement (66\%), meniscal repair (95\%), and chondral procedures (79\%) were critical. When performing a meniscal debridement, determining which tears require meniscectomy (64\%), identifying the proper amount of meniscus to resect (63.3\%), and performing the meniscectomy (53\%) were all considered critical. Additionally, all aspects of performing a meniscal repair were critical (Table 2). For chondral procedures, microfracture was deemed critical (76\%) while performing a chondroplasty was not (47\%). 


\section{Cureus}

Overall Knee Arthroscopy Critical Elements, n (\%), N=343

Establishing Portals

154 (44.9)

Diagnostic Arthroscopy

166 (48.4)

Meniscal Debridement

$225(65.6)$ *

Meniscal Repair

$327(95.3)$ *

Chondral Procedures

$271(79.0)$ *

Closure

$12(3.5)$

None

Positioning

47 (13.7)

Knee Arthroscopy Critical Establishing Portal Elements, n (\%), N=343

Anterior Portals

$141(41.1)$

Posterior Portals for Meniscal Root Repair

Knee Arthroscopy Critical Meniscal Debridement Elements, n (\%), N=343

Identifying which Tears Need Meniscectomy vs Repair

Identifying Amount of Meniscal Debridement

Performing Meniscectomy with Shaver and/or Biter

Knee Arthroscopy Critical Meniscal Repair Elements, n (\%), N=343

Establishing Technique (All inside/Outside In/ Inside Out)

Approach if using Outside $\ln /$ Inside Out

$268(78.1)$ *

Identifying Location of Suture Placement

$282(82.2)$ *

All-Inside Repair

$296(86.3)$ *

Passing Sutures for Outside-in or Inside-out

$287(83.7)$ *

Tying Knots

$204(59.5)$ *

Knee Arthroscopy Critical Chondral Procedure Elements, n (\%), N=343

Microfracture

$260(75.8)$ *

Chondroplasty

$161(46.9)$

\section{TABLE 2: Critical steps of knee arthroscopy}

(*) signifies a critical element.

For ACL reconstruction, all steps were critical except preparing the footprints, closing, and positioning (Table 3). With regard to autograft harvesting during ACL reconstruction, identifying (65\%) and stripping the hamstring tendons (69\%), selecting the size of bone-patella-bone (BTB) graft (63\%), and harvesting the BTB graft with a microsagittal saw (81\%) were critical steps, but preparation of the graft on the back table was not (20\%). Performing a notchplasty and debriding the ACL ligament were not considered critical steps. Regarding tunnel placement, identifying the proper location (95\%), holding the drill guide (68\%), and drilling the tunnels (61\%) were critical steps. Lastly, the steps of graft fixation were all critical, except for cycling the graft. This included shuttling the graft, determining the appropriate graft tension, and securing the graft with interference screws or aperture fixation. 


\section{Cureus}

Overall ACL Reconstruction Critical Elements, $n(\%), N=343$

Establishing Portals

$180(52.5)$ *

Graft Harvest

$300(87.5)$ *

Diagnostic Arthroscopy

$179(52.2)$ *

Debridement/Preparation of Footprints

$189(55.1)$ *

Tunnel Placement

$335(97.7)$ *

Graft Fixation

$316(92.1)$ *

Closing Patella Tendon/Closure

$64(18.7)$

None

$1(0.3)$

Positioning

$96(28.0)$

ACL Reconstruction Critical Graft Harvest Elements, $n(\%), N=343$

Surgical Approach

$166(48.4)$

Identifying Hamstring Tendons

$224(65.3)$ *

Stripping Tendons with Tendon Stripper

$237(69.1)$ *

BTB - Selecting Size of Patella Graft

$215(62.7)$ *

BTB - Using Microsagittal Saw to Harvest Bone Plugs

$277(80.8)$ *

Preparing Graft on Back Table

67 (19.5)

ACL Reconstruction Critical Debridement/Footprint Preparation Elements, $n$ (\%), N=343

Notchplasty

Debriding Footprints/ Removing ACL Stump

149 (43.4)

ACL Reconstruction Critical Tunnel Placement Elements, $n(\%), \mathrm{N}=343$

Identifying Tunnel Position

$324(94.5)$ *

Holding Guide for Guide Pins

$232(67.6)$ *

Drilling Tunnels

$210(61.2)$ *

ACL Reconstruction Critical Graft Fixation Elements, $n(\%), ~ N=343$

Graft Passage/Shuttling

$249(72.6)$ *

Cycling Graft

$139(40.5)$

Determining Appropriate Graft Tension

$260(75.8)$ *

Placing Interference Screws / Aperture Fixation

$298(86.9)$ *

\section{TABLE 3: Critical steps of ACL reconstruction}

ACL - anterior cruciate ligament; BTB - bone-patella-bone

(*) signifies a critical element.

For rotator cuff repair, establishing portals (54\%), preparing the footprint (64\%), and repairing the tendon (94\%) were critical. Positioning, diagnostic arthroscopy, subacromial bursectomy, and closure were found to be non-critical steps (Table 4). Further evaluation found that all aspects of repairing the tendon were critical, including identifying suture placement, passing sutures, identifying the number and location of anchors, determining single versus double row, and tying suture knots. 


\section{Cureus}

Overall Rotator Cuff Repair Critical Elements, n (\%), N=343

Establishing Portals

$185(53.9)$ *

Diagnostic Arthroscopy

$171(49.9)$

Subacromial Bursectomy

149 (43.4)

Preparing Footprint

$221(64.4)$ *

Repairing Tendon

$323(94.2)$ *

Closure

$13(3.8)$

None

$2(0.6)$

Positioning

$96(28.0)$

Rotator Cuff Repair Critical Establishing Portal Elements, $n$ (\%), N=343

Portals in Lateral Decubitus Position

$152(44.3)$

Portals in Beach Chair Position

$133(38.8)$

Rotator Cuff Repair Critical Subacromial Bursectomy Elements, n (\%), N=343

Bursectomy

129 (37.6)

Acromioplasty

Tear Assessment

$147(42.9)$

Rotator Cuff Repair Critical Footprint Preparation Elements, n (\%), N=343

Using Shaver/Burr to Decorticate Humeral Footprint

$176(51.3)$ *

Debriding Tendon Edges

$163(47.5)$

Tendon Mobilization

$215(62.7)$ *

Marginal Convergence

$217(63.3)$ *

Interval Slide

$206(60.1)$ *

Rotator Cuff Repair Critical Tendon Repair Elements, n (\%), N=343

Identifying Location of Suture Placement

$298(86.9)$ *

Passing Sutures with Suture Passer

$261(76.1)$ *

Passing Sutures with Suture Lasso

$212(61.8)$ *

Identifying Number of Anchors

$285(83.1)$ *

Identifying Location of Anchors

$306(89.2)$ *

Determining Singer vs Double Row

$274(79.9)$ *

Tying Suture Knots

$258(75.2)$ *

\section{TABLE 4: Critical steps of rotator cuff repair}

(*) signifies a critical element.

For arthroscopic anterior capsulorrhaphy with labral repair (or Bankart procedure), the critical elements were the establishment of portals (64\%), glenoid preparation (80\%), passing sutures (90\%), and placing anchors (93\%) (Table 5). Within glenoid preparation, both the mobilization of the labrum and decorticating the glenoid neck were critical. Passing sutures, the selection of the number and placement of anchors, drilling for anchors, and tying suture knots were all found to be critical. Similar to arthroscopic rotator cuff repair, diagnostic arthroscopy and closure were not critical. 


\section{Cureus}

Overall Capsulorrhaphy, Anterior; with Labral Repair (i.e. Bankart Procedure) Critical Elements, n (\%), N=343

Establishing Portals

$221(64.4)$ *

Diagnostic Arthroscopy

$169(49.3)$

Preparing Glenoid

$274(79.9)$ *

Passing Sutures

$309(90.1)$ *

Placing Anchors

$320(93.3)^{*}$

Closure

$6(1.7)$

Positioning

107 (31.2)

Bankart Repair Critical Establishing Portals Elements, $n$ (\%), N=343

Portals in Lateral Decubitus Position

$185(53.9)$ *

Portals in Beach Chair Position

$139(40.5)$

Bankart Repair Critical Glenoid Preparation Elements, $n$ (\%), N=343

Mobilizing Labrum

$271(79.0)$ *

Decorticating Glenoid Neck

$240(70.0)$ *

Bankart Repair Critical Passing Sutures Elements, $n(\%), N=343$

Passing Sutures at 3 o'clock Position

$225(65.6)$ *

Passing Sutures at 5-6 o'clock Position

$307(89.5)$ *

Bankart Repair Critical Placing Anchors Elements, $n$ (\%), N=343

Selecting Number of Anchors

$276(80.5)$ *

Selecting Location of Anchors

$308(89.8)$ *

Using Knotless Anchors

$226(65.9)$ *

Using Traditional Anchors

$228(66.5)$ *

Drilling Anchors

$274(79.9)$ *

Tying Suture Knots

$255(74.3)$ *

TABLE 5: Critical steps of anterior capsulorrhaphy with labral repair (Bankart procedure)

(*) signifies a critical element.

\section{Private versus academic}

For knee arthroscopy, private practice surgeons rated all aspects of knee arthroscopy except closure and positioning to be critical. Academic surgeons, on the other hand, did not find portal establishment $(\mathrm{p}<0.0001)$ or diagnostic arthroscopy $(\mathrm{p}<0.0001)$ to be critical. With regards to chondral procedures, private surgeons found chondroplasty to be a critical element while academic surgeons did not $(\mathrm{p}=0.007)$. For ACL reconstruction, private surgeons rated portal establishment $(\mathrm{p}<0.0001)$, diagnostic arthroscopy $(\mathrm{p}<0.0001)$, and debridement of footprint/ACL $(\mathrm{p}=0.012$ ) to be critical while academic surgeons did not.

For RCR, private surgeons rated portal establishment ( $\mathrm{p}<0.0001)$, diagnostic arthroscopy ( $<<0.0001)$, and subacromial decompression $(\mathrm{p}=0.006$ ) as critical steps while academic surgeons did not. For the Bankart procedure, private surgeons rated diagnostic arthroscopy $(\mathrm{p}<0.0001)$ and portal establishment $(\mathrm{p}<0.0001)$ as critical while academic surgeons did not (Table 6 ).

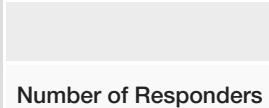

Number of Responders
Academic

83

Private

P-value

183 


\section{Cureus}

Overall ACL Reconstruction Critical Elements, n (\%), N=266

Establishing Portals

28 (33.7)

$121(66.1)$

$<0.0001$

Diagnostic Arthroscopy

$28(33.7)$

$122(66.7)$

$<0.0001$

Debridement/Preparation of Footprints

40 (48.2)

118 (64.5)

0.012

Closing Patella Tendon/Closure

$4(4.8)$

$44(24.0)$

$<0.0001$

Positioning

17 (20.5)

62 (33.9)

0.027

ACL Reconstruction Critical Graft Harvest Elements, $n$ (\%), $N=266$

Surgical Approach

27 (32.5)

$110(60.1)$

$<0.0001$

ACL Reconstruction Critical Debridement/Footprint Preparation Elements, n (\%), N=266

Notchplasty

28 (33.7)

89 (48.6)

0.023

Debriding Footprints/ Removing ACL Stump

$31(37.3)$

$94(51.4)$

0.034

ACL Reconstruction Critical Tunnel Placement Elements, $n$ (\%), N=266

Identifying Tunnel Position

75 (90.4)

$178(97.3)$

0.027

Overall Knee Arthroscopy Critical Elements, n (\%), N=266

Establishing Portals

$20(24.1)$

$102(55.7)$

$<0.0001$

Diagnostic Arthroscopy

$24(28.9)$

109 (59.6)

$<0.0001$

Knee Arthroscopy Critical Establishing Portal Elements, n (\%), N=266

Anterior Portals

$18(21.7)$

$96(52.5)$

$<0.0001$

Posterior Portals for Meniscal Root Repair

$<0.0001$

Knee Arthroscopy Critical Chondral Procedure Elements, n (\%), N=266

Chondroplasty

31 (37.3)

$101(55.2)$

0.007

Overall Rotator Cuff Repair Critical Elements, $n$ (\%), N=266

Establishing Portals

27 (32.5)

$123(67.2)$

$<0.0001$

Diagnostic Arthroscopy

23 (27.7)

$114(62.3)$

$<0.0001$

Subacromial Bursectomy

28 (33.7)

95 (51.9)

0.006

Positioning

15 (18.1)

62 (33.9)

0.008

Rotator Cuff Repair Critical Establishing Portal Elements, n (\%), N=266

Portals in Lateral Decubitus Position

24 (28.9)

$102(55.7)$

$<0.0001$

Portals in Beach Chair Position

$20(24.1)$

$87(47.5)$

$<0.0001$

Rotator Cuff Repair Critical Subacromial Bursectomy Elements, n (\%), N=266

Bursectomy

$20(24.1)$

89 (48.6)

$<0.0001$

Acromioplasty

$25(30.1)$

$93(50.8)$

0.002

Tear Assessment

$28(33.7)$

$93(50.8)$

0.01

Rotator Cuff Repair Critical Footprint Preparation Elements, n (\%), N=266

Using Shaver/Burr to Decorticate Humeral Footprint

$34(41.0)$

108 (59.0)

0.006

Debriding Tendon Edges

32 (38.6)

100 (54.6)

0.015

Overall Capsulorrhaphy, Anterior; with Labral Repair (i.e. Bankart Procedure) Critical Elements, n (\%), N=266

Establishing Portals

$39(47.0)$

$135(73.8)$

$<0.0001$

Diagnostic Arthroscopy

24 (28.9)

112 (61.2)

$<0.0001$

Bankart Repair Critical Establishing Portals Elements, n (\%), N=266 


\section{Cureus}

TABLE 6: Differences according to setting (academic vs. private)

$\mathrm{ACL}$ - anterior cruciate ligament

(*) signifies a critical element.

\section{Years in practice}

Based on years of experience, surgeons with less experience ( $<15$ years) did not rate portal establishment, diagnostic arthroscopy, or ligament debridement/notchplasty as critical ACL reconstruction steps while older surgeons did. Similar results were obtained for knee arthroscopy, with portal establishment and diagnostic arthroscopy regarded as non-critical by younger surgeons but critical by those with greater than 15 years of experience. Surgeons with less experience did not regard chondroplasty and meniscal debridement as critical, while the more experienced surgeons $\operatorname{did}(\mathrm{p}=0.032, \mathrm{p}=0.004$, respectively). For RCR, experienced surgeons considered decorticating footprint $(\mathrm{p}=0.38)$ and debriding tendon edges $(\mathrm{p}=0.183)$ to be critical while less experienced surgeons did not, however, the difference did not meet significance. All surgeons regardless of experience considered every aspect of the tendon repair (passing sutures, identifying the location of anchors, placing anchors, tying knots) to be critical. When comparing across surgeons for the Bankart procedure, closure and patient positioning were similarly regarded as non-critical aspects of the procedure. Younger surgeons, however, did not consider diagnostic arthroscopy to be critical (47\% vs. $50 \%$, $\mathrm{p}=0.535$ ). Both cohorts regarded all sub-steps of this procedure to be critical and there were no differences between them.

\section{First assistant}

When comparing across years in practice, there was no difference in first assistant utilization. First assistants included residents, physician assistants (PAs), and nurse practitioners (NPs). Unlike surgeons with a resident as first assist, surgeons with a PA or NP found every step of each procedure to be critical except closure and positioning (Table 7). For all procedures, when a resident was first assistant, positioning, portal establishment, diagnostic arthroscopy, and closure were not found to be critical. For ACL reconstruction, when a resident was the first assistant, the only critical steps were graft harvest, tunnel placement, and graft fixation. For knee arthroscopy, chondroplasty was not found to be critical when a resident was first assistant. For RCR, tendon repair was the only critical step.

\begin{tabular}{|c|c|c|c|}
\hline & Resident First Assist & PA/NP & P-value \\
\hline Number of Responders & 102 & 169 & - \\
\hline \multicolumn{4}{|c|}{ Overall ACL Reconstruction Critical Elements, $n(\%), \mathrm{N}=343$} \\
\hline Establishing Portals & $33(32.4)$ & $105(61.8)$ & $<0.0001$ \\
\hline Diagnostic Arthroscopy & $30(29.4)$ & $110(64.7)$ & $<0.0001$ \\
\hline Debridement/Preparation of Footprints & 39 (38.2) & $110(64.7)$ & $<0.0001$ \\
\hline Closing Patella Tendon/Closure & $6(5.9)$ & $35(20.6)$ & 0.001 \\
\hline \multicolumn{4}{|c|}{ ACL Reconstruction Critical Graft Harvest Elements, $n(\%), \mathrm{N}=272$} \\
\hline Surgical Approach & 29 (28.4) & $100(58.8)$ & $<0.0001$ \\
\hline \multicolumn{4}{|c|}{ ACL Reconstruction Critical Debridement/Footprint Preparation Elements, $n(\%), N=272$} \\
\hline Notchplasty & $23(22.5)$ & $88(51.8)$ & $<0.0001$ \\
\hline Debriding Footprints/ Removing ACL Stump & 29 (28.4) & 87 (51.2) & $<0.0001$ \\
\hline \multicolumn{4}{|c|}{ ACL Reconstruction Critical Tunnel Placement Elements, n (\%), N=272 } \\
\hline Identifying Tunnel Position & $91(89.2)$ & 169 (99.4) & $<0.0001$ \\
\hline \multicolumn{4}{|c|}{ Overall Knee Arthroscopy Critical Elements, n (\%), N=272 } \\
\hline Establishing Portals & 25 (24.5) & $93(54.7)$ & $<0.0001$ \\
\hline
\end{tabular}




\section{Cureus}

\begin{tabular}{|c|c|c|c|}
\hline Diagnostic Arthroscopy & $28(27.5)$ & $103(60.6)$ & $<0.0001$ \\
\hline Meniscal Debridement & $60(58.8)$ & $124(72.9)$ & 0.016 \\
\hline Positioning & $6(5.9)$ & $23(13.5)$ & 0.048 \\
\hline \multicolumn{4}{|c|}{ Knee Arthroscopy Critical Establishing Portal Elements, n (\%), N=272 } \\
\hline Anterior Portals & $20(19.6)$ & $87(51.2)$ & $<0.0001$ \\
\hline Posterior Portals for Meniscal Root Repair & $21(20.6)$ & $87(51.2)$ & $<0.0001$ \\
\hline \multicolumn{4}{|c|}{ Knee Arthroscopy Critical Meniscal Debridement Elements, n (\%), N=272 } \\
\hline Identifying which Tears Need Meniscectomy vs Repair & $56(54.9)$ & $122(71.8)$ & 0.005 \\
\hline Identifying Amount of Meniscal Debridement & $58(56.9)$ & $119(70.0)$ & 0.028 \\
\hline Performing Meniscectomy with Shaver and/or Biter & $41(40.2)$ & $103(60.6)$ & 0.001 \\
\hline \multicolumn{4}{|c|}{ Knee Arthroscopy Critical Meniscal Repair Elements, $n$ (\%), N=272 } \\
\hline Establishing Technique (All inside/Outside In/ Inside Out) & 77 (75.5) & $148(87.1)$ & 0.015 \\
\hline Approach if using Outside In/ Inside Out & 75 (73.5) & $143(84.1)$ & 0.034 \\
\hline Tying Knots & $54(52.9)$ & $111(65.3)$ & 0.043 \\
\hline \multicolumn{4}{|c|}{ Knee Arthroscopy Critical Chondral Procedure Elements, n (\%), N=272 } \\
\hline Chondroplasty & 35 (34.3) & $92(54.1)$ & 0.002 \\
\hline \multicolumn{4}{|l|}{ Overall Rotator Cuff Repair Critical Elements, n (\%), N=272 } \\
\hline Establishing Portals & $31(30.4)$ & $110(64.7)$ & $<0.0001$ \\
\hline Diagnostic Arthroscopy & 27 (26.5) & $108(63.5)$ & $<0.0001$ \\
\hline Subacromial Bursectomy & $28(27.5)$ & 91 (53.5) & $<0.0001$ \\
\hline Preparing Footprint & $57(55.9)$ & $118(69.4)$ & 0.024 \\
\hline \multicolumn{4}{|c|}{ Rotator Cuff Repair Critical Establishing Portal Elements, n (\%), N=272 } \\
\hline Portals in Lateral Decubitus Position & $23(22.5)$ & $92(54.1)$ & $<0.0001$ \\
\hline Portals in Beach Chair Position & $22(21.6)$ & 79 (46.5) & $<0.0001$ \\
\hline \multicolumn{4}{|c|}{ Rotator Cuff Repair Critical Subacromial Bursectomy Elements, $n(\%), \mathrm{N}=272$} \\
\hline Bursectomy & $18(17.6)$ & $83(48.8)$ & $<0.0001$ \\
\hline Acromioplasty & $23(22.5)$ & $90(52.9)$ & $<0.0001$ \\
\hline Tear Assessment & $28(27.5)$ & $89(52.4)$ & $<0.0001$ \\
\hline \multicolumn{4}{|c|}{ Rotator Cuff Repair Critical Footprint Preparation Elements, n (\%), N=272 } \\
\hline Using Shaver/Burr to Decorticate Humeral Footprint & 39 (38.2) & $98(57.6)$ & 0.002 \\
\hline Debriding Tendon Edges & $36(35.3)$ & $90(52.9)$ & 0.005 \\
\hline Tendong Mobilization & 55 (53.9) & $115(67.6)$ & 0.024 \\
\hline Marginal Convergence & $55(53.9)$ & $116(68.2)$ & 0.018 \\
\hline Interval Slide & $51(50.0)$ & $112(65.9)$ & 0.01 \\
\hline \multicolumn{4}{|c|}{ Rotator Cuff Repair Critical Tendon Repair Elements, n (\%), N=272 } \\
\hline Identifying Location of Suture Placement & $84(82.4)$ & $154(90.6)$ & 0.047 \\
\hline Identifying Location of Anchors & $88(86.3)$ & $160(94.1)$ & 0.027 \\
\hline \multicolumn{4}{|c|}{ Overall Capsulorrhaphy, Anterior; with Labral Repair (i.e. Bankart Procedure) Critical Elements, n (\%), N=272 } \\
\hline Establishing Portals & $50(49.0)$ & $124(72.9)$ & $<0.0001$ \\
\hline Diagnostic Arthroscopy & $26(25.5)$ & $108(63.5)$ & $<0.0001$ \\
\hline
\end{tabular}




\section{Cureus}

Bankart Repair Critical Establishing Portals Elements, n (\%), N=272

Portals in Lateral Decubitus Position

$108(63.5)$

$<0.0001$

Portals in Beach Chair Position

$31(30.4)$

77 (45.3)

0.015

Bankart Repair Critical Glenoid Preparation Elements, $n$ (\%), N=272

Decorticating Glenoid Neck

$64(62.7)$

$128(75.3)$

0.028

\section{TABLE 7: Differences according to first assistant}

PA - physician assistant, NP - nurse practitioner, ACL - anterior cruciate ligament

\section{Discussion}

Every surgical procedure is a series of steps, some more critical than others. Some steps of a procedure are so important that any misstep or lack of guidance may expose the patient to undue harm. Despite their crucial role in surgical training, billing and the ethicality of concurrent surgery, the "critical steps" of arthroscopy procedures have not yet been clearly defined and interpretation has fallen in the hands of the surgeon. The current study aims to reach a greater consensus of which steps are generally deemed critical by surgeons and to identify how this may vary according to certain demographic parameters. The elucidation of critical elements for surgical procedures has potential ramifications on surgical education, concurrent surgery, surgical billing, and medical ethics.

Recent press reports have suggested that there is an increase in adverse patient outcomes and longer procedure times when an attending surgeon is operating in two different surgical suites [1]. The concept of concurrent surgeries in orthopedic literature is limited; however, a recent analysis of overlapping surgery in the ambulatory setting has been described. Zhang et al. performed a retrospective review over a three-year period and found that $68 \%$ of cases were concurrent while $32 \%$ were not [6]. They found no difference in the postoperative complication rate between the cohorts $(1.1 \%$ vs. $1.3 \%, \mathrm{p}=0.811)$. They also concluded that overlapping surgery yields an equivalent operating time in an ambulatory setting [6]. National registry data from the American College of Surgeons in greater than 20,000 knee and shoulder arthroscopic cases has shown these procedures to be inherently safe, with a 30 -day complication rate of $1.6 \%$ and $0.99 \%$, respectively [7-8].

We report that for the most common arthroscopic sports medicine surgeries, there was considerable variability in the elements deemed "critical" by the surveyed respondents. Patient positioning and closure were not deemed critical steps among all procedures. Diagnostic arthroscopy was only critical when performing ACL reconstruction. Interestingly, the establishment of arthroscopic portals was viewed as critical for all procedures except knee arthroscopy.

We found significant differences between academic and private practice surgeons, suggesting that the notion of "critical element" may be influenced by the surgical setting. Notably, nearly all steps in all four procedures were considered critical by private practice surgeons. Often, surgeons practicing in a private setting lack highly trained surgical assistants, such as residents and fellows, who are qualified and capable of performing a number of operative steps independently. As a result, nearly every step of a surgical procedure in a private practice requires the direct supervision of the surgeon.

Younger surgeons, defined as those with less than 15 years of practice, were significantly less likely to consider diagnostic arthroscopy and portal establishment as critical steps of the procedure. The difference is likely, in part, due to a comfort level with arthroscopy cases since nearly $98 \%$ of the younger respondents had completed a sports medicine fellowship compared to only $82 \%$ of the more experienced surgeons $(\mathrm{p}<0.01)$.

Although completely novel in the sports medicine literature, there are limitations to the current study. First, the study is limited by the response rate. The survey was distributed to over 2,000 surgeons; however, only $17 \%$ responded to the survey. One reason for an imperfect response rate may be that there is no way of identifying how many surgeons received the email. Some of these emails may have been processed as spam or the email listed is not the primary email used by the surgeon. Overall, establishing a consensus regarding the "critical" elements of surgery may necessitate a more robust sample size. Lastly, a potential limitation is that there are likely other factors that influence how surgeons classify different steps of surgery. Patient characteristics, including body weight, comorbid conditions, anatomic variations, and prior arthroscopic procedures, may significantly influence a surgeon's perception of the surgical procedure.

\section{Conclusions}


The notion of "critical" is used colloquially by the medical community in the form of national guidelines, consent forms, and reimbursement regulations. Until now, there have not been any attempts to define critical steps of arthroscopic sports medicine procedures. For four of the most common arthroscopic sports medicine procedures, elements that were not regarded as critical routinely included positioning, the establishment of portals, and closure. However, given the variability based on surgical subspecialty, surgical setting, and surgeon experience, it is difficult to reach a general consensus and standardized definitions of "critical" elements should be established by professional sports medicine societies.

\section{Additional Information \\ Disclosures}

Human subjects: Consent was obtained by all participants in this study. Animal subjects: All authors have confirmed that this study did not involve animal subjects or tissue. Conflicts of interest: In compliance with the ICMJE uniform disclosure form, all authors declare the following: Payment/services info: All authors have declared that no financial support was received from any organization for the submitted work. Financial relationships: All authors have declared that they have no financial relationships at present or within the previous three years with any organizations that might have an interest in the submitted work. Other relationships: All authors have declared that there are no other relationships or activities that could appear to have influenced the submitted work.

\section{References}

1. Boston Globe S report. Clash in the name of care. (2012). Accessed: October 10, 2018: https://apps.bostonglobe.com/spotlight/clash-in-the-name-of-care/story/ .

2. Department of Health \& Human Services (DHHS). Centers for Medicare \& Medicaid Services (CMS). Transmittal 2303. (2011). Accessed: October 10, 2018: http://www.cms.gov/Regulations-andGuidance/Guidance/Transmittals/downloads/R2303CP.pdf.

3. Our view of overlapping surgery. (2016). Accessed: October 10, 2018: https://www.bostonglobe.com/opinion/editorials/2016/01/10/our-view-overlappingsurgery/m0h9GxMsEzziBRWAVhZlvK/story.html.

4. Statements on principles. American College of Surgeons . (2016). Accessed: October 10, 2018: https://www.facs.org/about-acs/statements/stonprin.

5. Beasley GM, Pappas TN, Kirk AD: Procedure delegation by attending surgeons performing concurrent operations in academic medical centers balancing safety and efficiency. Ann Surg. 2015, 261:1044-1045. 10.1097/SLA.0000000000001208

6. Zhang AL, Sing DC, Dang DY, Ma CB, Black D, Vail TP, Feeley BT: Overlapping surgery in the ambulatory orthopaedic setting. J Bone Joint Surg Am. 2016, 16:1859-1867. 10.2106/JBJS.16.00248

7. Martin CT, Pugely AJ, Gao Y, Wolf BR: Risk factors for thirty-day morbidity and mortality following knee arthroscopy: a review of 12,271 patients from the National Surgical Quality Improvement Program database. J Bone Joint Surg Am. 2013, 17:1-10.

8. Martin CT, Gao Y, Pugely AJ, Wolf BR: 30-day morbidity and mortality after elective shoulder arthroscopy: a review of 9410 cases. J Shoulder Elbow Surg. 2013, 22:1667-1675. 10.1016/j.jse.2013.06.022 